\title{
Isolation of Nanocellulose from Cotton Cellulose and Computer Modeling of Its Structure
}

\author{
Abdumutolib Atakhanov, Islom Turdikulov, Burhon Mamadiyorov, \\ Nodira Abdullaeva, Ilnar Nurgaliev, Yunusov Khaydar, Sayora Rashidova \\ Institute of Polymer Chemistry and Physics, Uzbekistan Academy of Sciences, Tashkent, Uzbekistan \\ Email: polymer@academy.uz, a-atakh?nov@yandex.ru
}

How to cite this paper: Atakhanov, A., Turdikulov, I., Mamadiyorov, B., Abdullaeva, N., Nurgaliev, I., Khaydar, Y. and Rashidova, S. (2019) Isolation of Nanocellulose from Cotton Cellulose and Computer Modeling of Its Structure. Open Journal of Polymer Chemistry, 9, 117-129.

https://doi.org/10.4236/ojpchem.2019.94010

Received: August 16, 2019

Accepted: November 2, 2019

Published: November 5, 2019

Copyright $\odot 2019$ by author(s) and Scientific Research Publishing Inc. This work is licensed under the Creative Commons Attribution International License (CC BY 4.0).

http://creativecommons.org/licenses/by/4.0/

\begin{abstract}
Nanocellulose is a new class of derivatives of cellulose, which is characterized by high crystallinity, surface area, degree of dispersion, ability to decomposition by microorganisms and etc. There is high attention solving problems of obtaining nanocellulose and its application as high quality filler for polymers, biodegradable materials, additives for papers, clotting dispersion and etc. Obtaining of particles of nanosized nanostructure on the base cellulose, studying of processes of their formation, properties and creation nanotechnology on this basis give the chance to obtain materials with unique properties. In this work nanocellulose was obtained from cotton cellulose by hydrolysis with sulfuric acid, ultrasonic dispersion and microwave irradiation. The properties and structure of nanocellulose are investigated by AFM, IR-spectroscopic, X-ray methods. Nanocellulose has rod-like shape with sizes $50-300 \mathrm{~nm}$ in length and $10-40 \mathrm{~nm}$ in diameters and spherical shape with sizes $50-300 \mathrm{~nm}$ depending on the synthesis conditions of obtaining. Quantum-chemical methods have been used to calculate the electronic characteristics of nanocellulose; the change in the energy difference between HOMO and LUMO is shown, showing the change in reactivity and the manifestation of specific properties.
\end{abstract}

\section{Keywords}

Nanocellulose, Cotton Cellulose, Acid Hydrolysis, Properties, Structure, Degree of Crystallinity, Quantum-Chemical Method

\section{Introduction}

Nanocellulose (NC) is a new class of derivatives of cellulose, which is characterized by high crystallinity and surface area, and ability to degradation by micro- 
organisms [1]. NC can be used as a high quality filler for polymers, additives for papers, clotting dispersion etc. [2] [3] [4]. In addition, their reaction activity increases due to the decrease of cellulose particles size, which allows the production of NC derivatives with new properties [5].

Nanocellulose and its derivatives present a new brand of nanotechnology that appears to have very wide applications in a variety of materials related domains where physical-chemical characteristics such as strength, weight, rheology and optical properties can be affected in a very positive manner [6].

Nanocellulose can be classified according to various factors such as shape, size and structure. For the shape characteristic, there are basically three types: sheet, spherical and whiskers. It is obvious that micro fibrillated nanocellulose (MFC) shape is sheet-like while microcrystalline cellulose exists in two forms: spherical and whisker. It should be noted that within each structure of nanocellulose there is a distribution of length, width, and percent crystallinity. These structures have several varieties such as (particle-like, rod-like, web-like and fiber-like). MFC has two forms: web-like and fiber-like, while NFC due to its structure has the form of fiber-like only [7] [8] [9].

A wide range of cellulose from plant raw materials: wood, bamboo, ambary, cellulose etc. are used as an initial material for obtaining $\mathrm{NC}$, and its geometrical characteristics, such as shape, particle size and composition (aldehyde and carboxyl groups) differ significantly depending on the source of raw materials and the obtaining conditions [10] [11] [12]. Decrease of the cellulose particle size until nanoscale is possible to achieve via various methods: mechanical treatment, acid hydrolysis, enzymatic hydrolysis and etc. [13] [14] [15].

Mechanism of obtaining NC usually is explained by acid hydrolysis of amorphous domains of cellulose, while the more persistent nanocrystallite remains intact and can be isolated in the form of rod-like particles. However, this mechanism does not explain why nanoparticles cannot be isolated even after intensive mechanical disintegration of cellulose hydrolyzing diluted acid solutions to limit the degree of polymerization [16]. The probable reason for this phenomenon is that the crystallites of cellulose are linked by strong intermolecular bonds.

Sufficient concentrated solution of acid can break these strong bonds and separate nanocrystallites. However, when cellulose hydrolyses with diluted solutions of acids, these bonds between the crystallites remain intact and nanoparticles of cellulose are formed instead of nanoparticles.

According to literature, the main properties of nanocellulose materials are: biodegradability, biocompatibility, high strength, high surface area, moisture absorption, dimensional stability and chemical functionality [14].

Hydrogen bonds, electrostatic interactions, Van der Waals interactions, donoracceptor interactions, hydrophilic-hydrophobic interactions, and $\pi$ - $\pi$-interactions are the main types of non-covalent interactions that are responsible for self-organization in biological systems. Evidence of complex charge transfers in the literature is known for solids and solutions in other areas of chemistry. The main 
electronic parameters associated with the orbitals in the molecule are the highest occupied molecular orbital (HOMO) and the lower free molecular orbital (LUMO) (energy and symmetry) can give us an idea of the main processes of proton and electron transfer in the excited state.

\section{Experimentation}

\subsection{Preparation of Cellulose and Nanocellulose}

Cotton cellulose with degree of polymerization (DP) 1200 was used for the preparations of NC. Cotton cellulose was obtained by cooking cotton linter in 1 $\mathrm{M}$ sodium hydroxyl solution at $120^{\circ} \mathrm{C}$ for 2 hours to remove hemicelluloses and lignin. It was washed with distilled water until the solution was neutral and bleached at $120^{\circ} \mathrm{C}$ for 2 hours using $4 \%$ hydrogen peroxide. The bleached fibers filtered and washed several times with distilled water and dried at $100^{\circ} \mathrm{C}$ for 4 hours.

Nanocellulose was isolated by acid hydrolysis. Acid hydrolysis of cotton cellulose was performed by slowly adding sulfuric acid under continuous stirring to a cellulose/water suspension placed in an ice water bath, until the final concentration of $50 \%-66 \% \mathrm{H}_{2} \mathrm{SO}_{4}$ was reached. The obtained suspension was then heated at different temperature $\left(25^{\circ} \mathrm{C}-45^{\circ} \mathrm{C}\right)$ for 0.5 - 2 hours. The suspension was constantly mixed using magnetic stirrer. The final suspensions were centrifuged using "D2012PLUS Ultracentrifuge" at room temperature for $20 \mathrm{mi}$ nutes with $8000 \mathrm{rpm}$ in order to remove the excess acid, then dialyzed in distilled water. Then the suspensions were treatment by microwave irradiation using equipment MDS-282 (frequency $2450 \mathrm{MHz}$ ), and ultrasonicated for an hour using GT SONIC-D3 ultrasonic bath. Afterwards, the suspensions were homogenized by using an ULTRA TURRAX Tube Drive homogenizer for 10 min.

\subsection{Characterization}

Structural and phase analyses of the samples were implemented using an X-ray diffractometer XRD-6100 (Shimadzu) with $\mathrm{Cu} \mathrm{K} \alpha$ radiation, generated at $60 \mathrm{kV}$ and $80 \mathrm{~mA}$ and step-scan mode $\left(2 \theta\right.$ range: $\left.0.1{ }^{\circ} \mathrm{C}-50^{\circ} \mathrm{C}\right)$. The crystalline index of cellulose was calculated using the Segal's empirical method [17].

Atomic force microscopy observations were carried out using Agilent 5500 . After a short sonication to prevent the formation of aggregates, drops of dilute cellulose suspensions were deposited onto freshly cleaved mica. After some time, the excess liquid was removed and the remaining film allowed to dry, then it was measured.

The FTIR spectra of nanocellulose were measured in the range from 4000 to $400 \mathrm{~cm}^{-1}$ (IRTracer-100, Shimadzu, Japan). The average DP was measured by the viscosity method using diluted solutions of cellulose powders in Schweizer's Reagent.

Quantum-chemical and semi-empirical methods in the framework of Hyper- 
chem programs, as well as ChemDraw and Chem3DPro, included in the package ChemBioOffice 2010 (CambridgeSoft) were used in studies

\section{Results and Discussion}

The study of the influence of temperature and hydrolysis time on the DC showed that the dependence is directly proportional, i.e. with increasing temperature $\left(25^{\circ} \mathrm{C}-45^{\circ} \mathrm{C}\right)$ and duration of hydrolysis $(0.5-2 \mathrm{~h})$ the DC of the obtained samples are increased $(79 \%-84 \%)$ (Table 1$)$.

However, it should be noted that the strong conditions of hydrolysis not only lead to a decrease in particle size, but also affects negatively the appearance quality of the final product. Therefore, the concentration of sulfuric acid of $61 \%$ at temperature $45^{\circ} \mathrm{C}$ for an hour formed a white suspension and the yield of which amounts up to $58 \%$. Color of the suspension becomes yellow with yield of $35 \%$ under the temperature $45^{\circ} \mathrm{C}$. With increasing temperature up to $60^{\circ} \mathrm{C}$ in the first 10 minutes there is a yellowing of the suspension, then after $1 \mathrm{~h}$ suspension becomes black, i.e., charring of the cellulose happens under these conditions. It was found that the optimum temperature for acid hydrolysis is $40^{\circ} \mathrm{C}-45^{\circ} \mathrm{C}$ and the optimal ratio for cellulose:acid solution $=1: 10$.

IR-spectroscopic studies show that the peaks of the typical cotton cellulose characterize NC; however, the intensity of peaks is higher (See Figure 1).

Research of influence of conditions for obtaining NC also showed that with the tightening of the conditions of hydrolysis process of removal of amorphous parts and crystalline parts occur due to dissolution of low molecular weight

Table 1. Degree of crystallite and sizes nanocellulose obtained at different temperature hydrolyzing process.

\begin{tabular}{|c|c|c|c|}
\hline \multirow{2}{*}{ Parameter } & \multicolumn{3}{|c|}{ Samples of NC obtained at temperature } \\
\hline & $25^{\circ} \mathrm{C}$ & $35^{\circ} \mathrm{C}$ & $45^{\circ} \mathrm{C}$ \\
\hline Degree of crystallite, $\%$ & 79 & 81 & 84 \\
\hline Sizes, nm & 230 & 180 & 120 \\
\hline
\end{tabular}

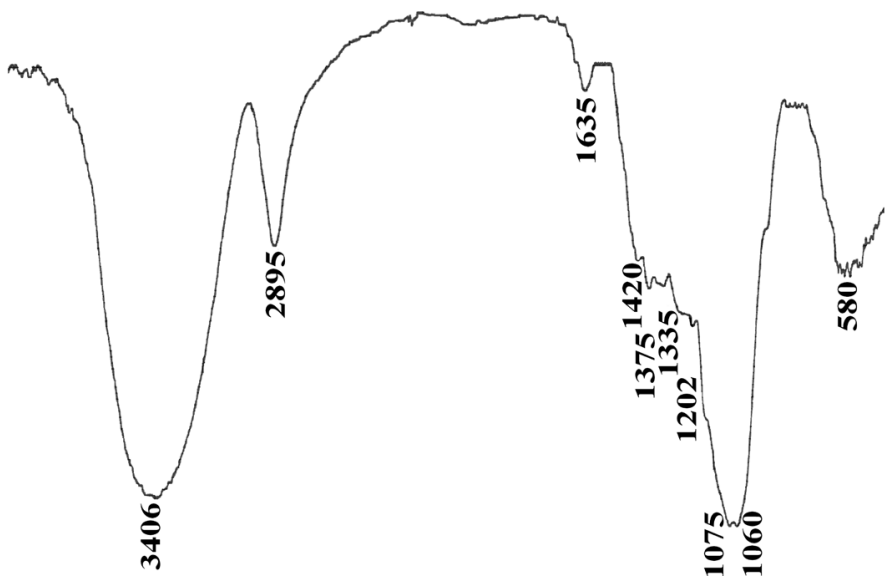

Figure 1. FTIR spectra of nanocellulose. 
fractions accompanied by a fall in the degree of polymerization and the yield of the final product.

The results showed the possibility of obtaining nanosized particles of cellulose $(<500 \mathrm{~nm})$ at a concentration of hydrolyzing agent more than $60 \%$. However, with increasing acid concentration, the average particle size decreases and the loss is increased due to the transition of cellulose into the solution. With further increase in acid concentration more than $66 \%$ there is a continued particle size reduction to the nano range and that product finally leads to the transition of cellulose into the solution and the solution becomes transparent.

According to XRD investigations, in the range of $\mathrm{H}_{2} \mathrm{SO}_{4}$ concentration from $50 \%$ to $61 \%$ the crystallinity of NC increases, further increase in acid concentration leads to a sharp decrease in the degree of crystallinity (DC) up to $25 \%$ (Figure 2(b)). Likely, the rupture of intermolecular hydrogen bonds between the cellulose fibrils, which leads to the destruction of the crystallites of cellulose and greater part in the solution, begins at high concentrations. Obtained NC particles kept $75 \%-81 \%$ crystallinity in range of concentration of $\mathrm{H}_{2} \mathrm{SO}_{4} 40 \%-62 \%$ (Figure 2(a)).

XRD investigations have shown that $\mathrm{NC}$, at considered interval of angle dispersion against amorphous halo, has three crystal maximum. The most intensive maximum observed at value of angle $2 \theta=22.5^{\circ}-22.6^{\circ}$ treat to crystallographic reflex (002). At result of mathematical processing, it has been established that the narrowest peak 1 could be carried to linear part of macromolecules of cellulose, the peak 2 to the deformed (bent) molecules, and the wide peak 3 to

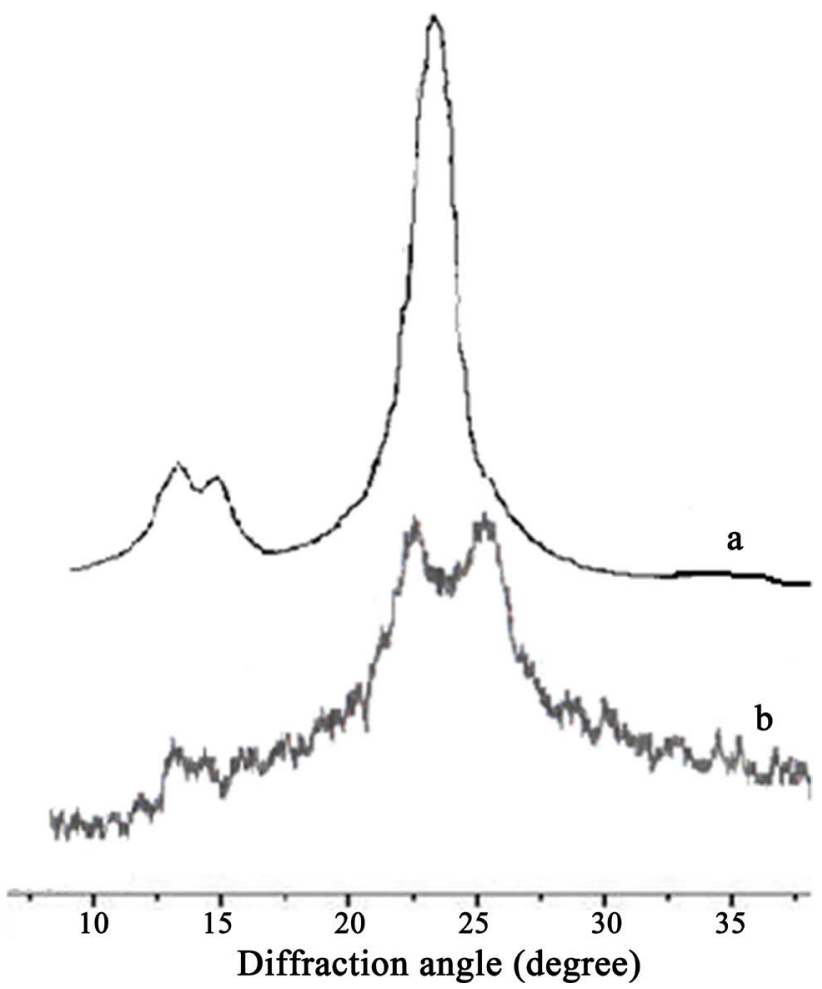

Figure 2. X-ray diffractograms of nanocellulose. 
amorphous cellulose.

Except specified maximum peaks are also observed partially over each other with indexes (101) and (10 $\overline{1})$ which arrange at angle $15^{\circ}-16.5^{\circ}$. Cristallophysical calculations give next sizes of interplanar spacing: $d_{101}=0.591 \mathrm{~nm}, d_{10 \overline{1}}=$ $0.537 \mathrm{~nm}, \mathrm{~d}_{002}=0.391 \mathrm{~nm}$ (Table 2). On the base of XRD diffraction width (10 $40 \mathrm{~nm}$ ) and length (50 - $300 \mathrm{~nm}$ ) elementary crystallites of NC were detected.

The NC, obtained at relatively low concentration of acid $<50 \%$ and temperatures $<50^{\circ} \mathrm{C}$ has rod-like structure (whiskers) with sizes $(50-300) \times(10-40)$ nm (Figure 3(a)). If the concentration of $\mathrm{H}_{2} \mathrm{SO}_{4}>50 \%$ and temperature is $>50^{\circ} \mathrm{C}$, then $\mathrm{NC}$ has ellipsoidal (spherical) forms (balls) with wide distribution in size (Figure $3(\mathrm{~b})$ ).

At increasing acid concentration process is accompanied by reduction of the sizes of particles with simultaneous depolymerization macromolecules of cellulose, at that intermolecular bonds between fibrils of cellulose start rupturing and formed particles move to hydrolyzing solution. The dissolved cellulose is precipitated from the solution with water cooling and has a low DC (less 30\%) and DP (less 80). In this case, perhaps, the assumption about the possibility of the process of reversion of monosaccharides, i.e. the reverse process of hydrolysis of polysaccharides can be assumed. The products of this process do not have linear form because the glucose molecule has four free hydroxyl groups, and interact with the terminal aldehyde groups of the glucose molecules in equal probability, which leads to the formation of highly branched structures. AFM studies revealed that the distribution of a large number of NC whiskers have a dimension of from 110 to $143 \mathrm{~nm}$, NC balls have $79-90 \mathrm{~nm}$.

Hydrogen bonds, electrostatic interactions, Van der Waals interactions, donoracceptor interactions, hydrophilic-hydrophobic interactions, and $\pi$ - $\pi$-interactions are the main types of non-covalent interactions that are responsible for self-organization in biological systems. The main electronic parameters associated with the orbitals in the molecule are the highest occupied molecular orbital (HOMO) and the lower free molecular orbital (LUMO) (energy and symmetry)

Table 2. Structural parameter of nanocellulose.

\begin{tabular}{|c|c|c|c|c|}
\hline \multirow{2}{*}{ Parameters } & \multicolumn{4}{|c|}{ Crystalline reflex } \\
\hline & 101 & $10 \overline{1}$ & 002 & 040 \\
\hline Location maximum $2 \theta$, (deg.) & 14.75 & 16.25 & 22.7 & 34.5 \\
\hline Interdimensional interval d, $(\AA)$ & 5.91 & 5.37 & 3.91 & 2.6 \\
\hline Width peak on 0.5 altitude $\beta$, (rad.) & 0.0209 & 0.0209 & 0.0235 & 0.0244 \\
\hline Size of crystalline particle $1,(\AA)$ & 67.71 & 67.83 & 60.90 & 60.21 \\
\hline Degree of crystallinity (on intensity 002 ) & \multicolumn{4}{|c|}{0.96} \\
\hline Degree of crystallinity (on area) & \multicolumn{4}{|c|}{0.81} \\
\hline Thickness amorphous seam, (̊) & \multicolumn{4}{|c|}{15.05} \\
\hline Big period L, $(\AA)$ & \multicolumn{4}{|c|}{79.21} \\
\hline
\end{tabular}




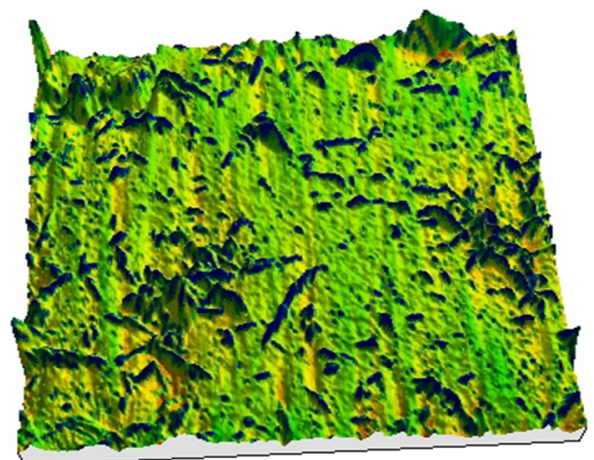

(a)

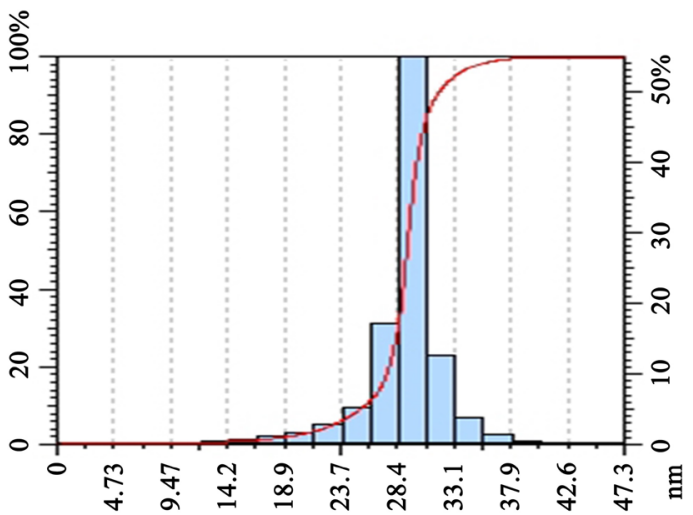

(a2)

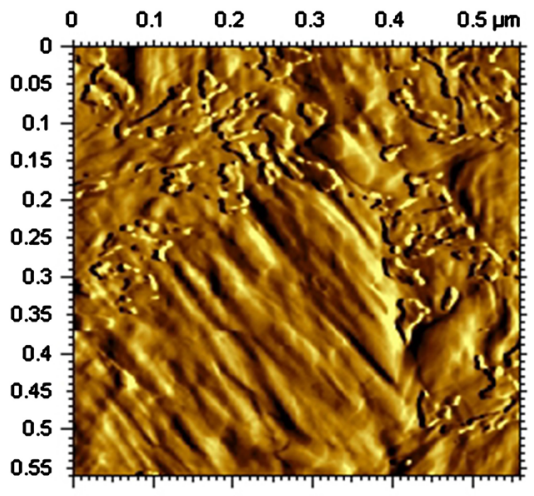

(b)

um

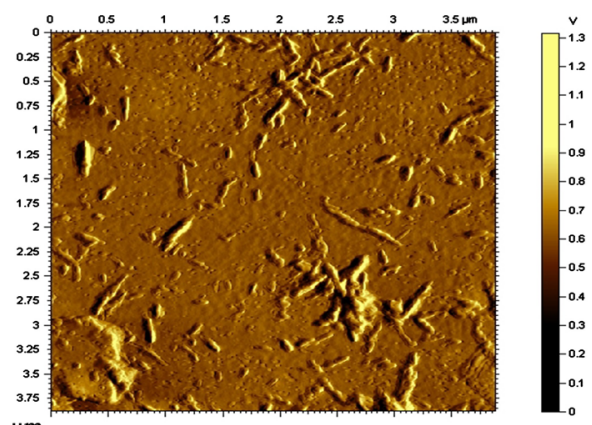

(a1)

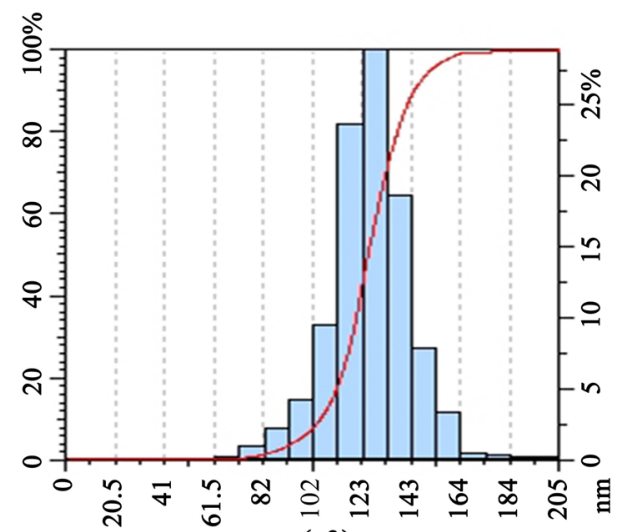

(a3)
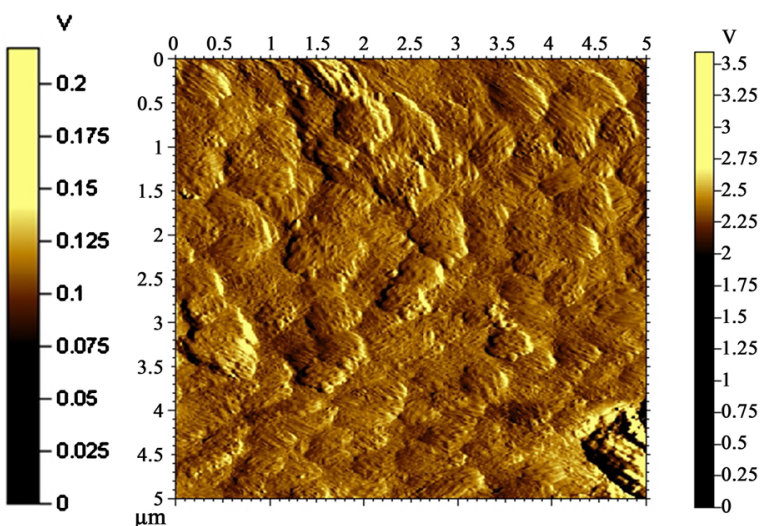

(b1)

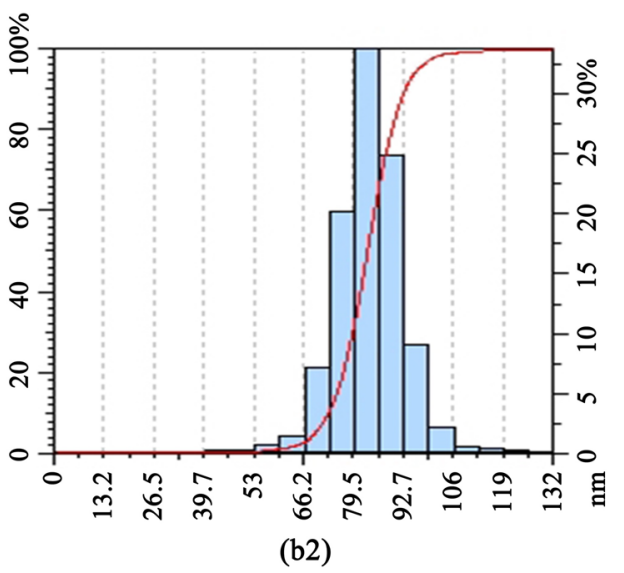

(b2)

Figure 3. AFM results of samples of rod-shape (a) and spherical (b) and their distribution on sizes. 
can give us an idea of the main processes of proton and electron transfer in the excited state.

It is known that electronic characteristics of substances differ in nanoscale. Models of cellulose chains consisting of 2 - 20 monomer units were built, with alternation for every 2 links (Figure 4). For all molecules, a complete optimization of the geometry was carried out by the method of molecular mechanics $(\mathrm{MM}+)$ and by the semi-empirical quantum chemical method PM3. Preliminary calculations were also carried out in the framework of the extended Hückel method and various semi-empirical methods of modifying the neglect of differential overlapping. However, these methods did not always produce even qualitatively correct results, and the convergence of the method was not always achieved. The AM1 method showed results close to the results of the PM3 method, but the convergence of the AM1 method is significantly worse than the convergence of the PM3 method.

The calculated HOMO and LUMO energies show the existence of interactions and, according to Mulliken's theory, the formation of complex charge transfers is associated with the transition of an electron from the HOMO orbitals of the donor to the LUMO of the acceptor orbital. However, the change in the difference of the energy values of HOMO and LUMO showed a non-monotonic character (Figure 5, Table 3), which indicates the distribution of the electron web
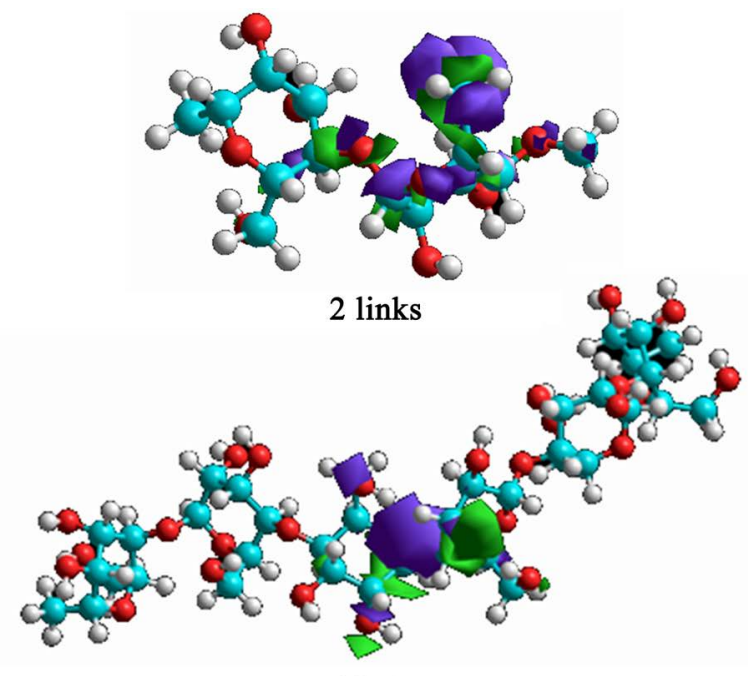

6 links

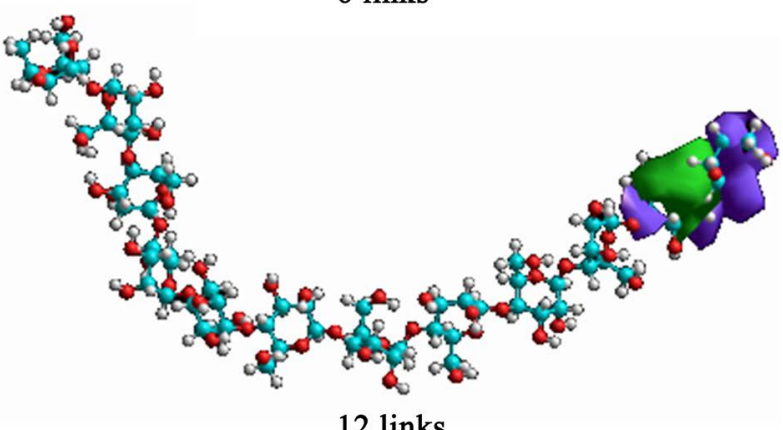

12 links

Figure 4. The electron density distribution depending on the size of the chain. 


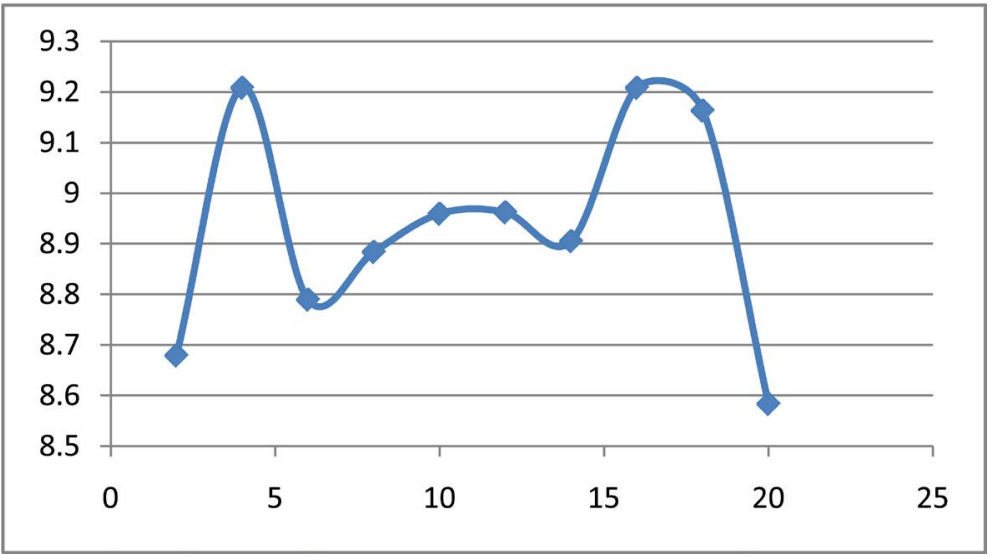

Figure 5. The change in $\triangle \mathrm{E}$ (HOMO-LUMO), $\mathrm{eV}$ of the number of cellulose units.

Table 3. Calculating the energy of cellulose molecules using the PM3 method.

\begin{tabular}{cc}
\hline Number of links & $\Delta \mathrm{E}\left(\mathrm{E}_{\text {Hомо }}-\mathrm{E}_{\mathrm{LUMO}}\right), \mathrm{eV}$ \\
\hline 2 & 8.679 \\
4 & 9.208 \\
6 & 8.789 \\
8 & 8.883 \\
10 & 8.959 \\
12 & 8.962 \\
14 & 8.905 \\
16 & 9.208 \\
18 & 9.163 \\
20 & 8.584 \\
\hline
\end{tabular}

along the entire length of the chain, but for each chain its change depends on the geometry of the structure and the number of formed intra-chain hydrogen bonds.

A quantum-chemical calculation of the models of possible end groups of nanocellulose obtained during hydrolysis was carried out (Figure 6). Since the presence of such groups changes the electronic characteristics, as a result, the reactivity changes and the specific properties of cellulose nanoparticles appear. The calculations were performed by the semi-empirical quantum-chemical extended Hukelya method.

The presence of functional groups of substituents and their number alter the electronic structure in such a way that leads to a narrowing of the energy gap between HOMO and LUMO orbitals, the numerical data of which can, in principle, be compared with the quantum-chemical index of the reactivity of Mulliken. The HOMO and LUMO orbitals are distributed in functional groups, which indicates a shift in the electron density to the most negative oxygen atoms and a further weakening of the glucose bond. The cellulose was inhabited (electron density) distributed over a 6-membered pyran cycle (Figure 7, Figure 8). 

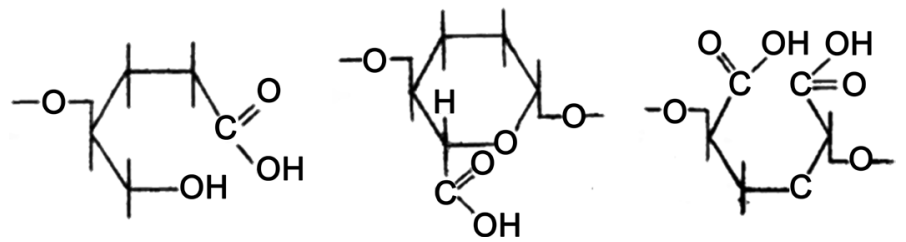

Figure 6. Possible options for changing the structure of cellulose during hydrolysis.
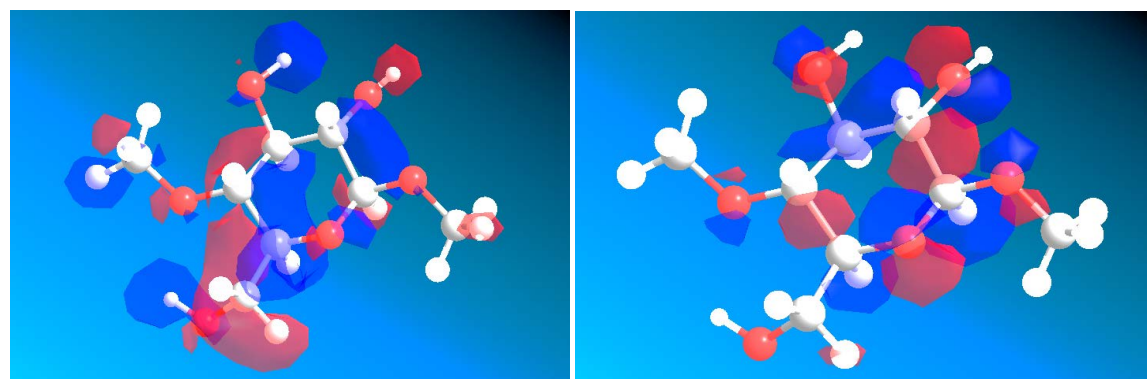

Figure 7. HOMO and LUMO orbitals of monomer chain of cellulose
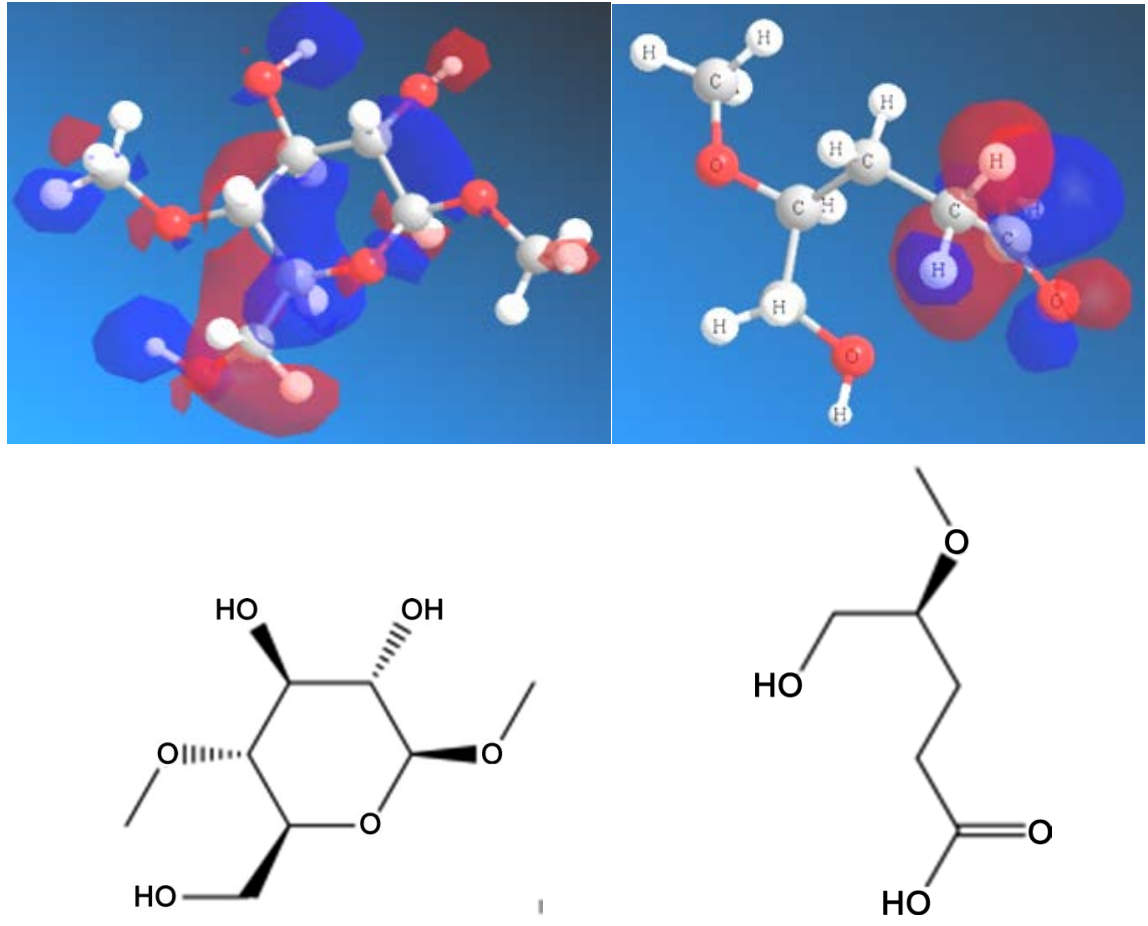

$\Delta \mathrm{E}=29,157 \mathrm{eV}$

$\Delta \mathrm{E}=12,537 \mathrm{eV}$
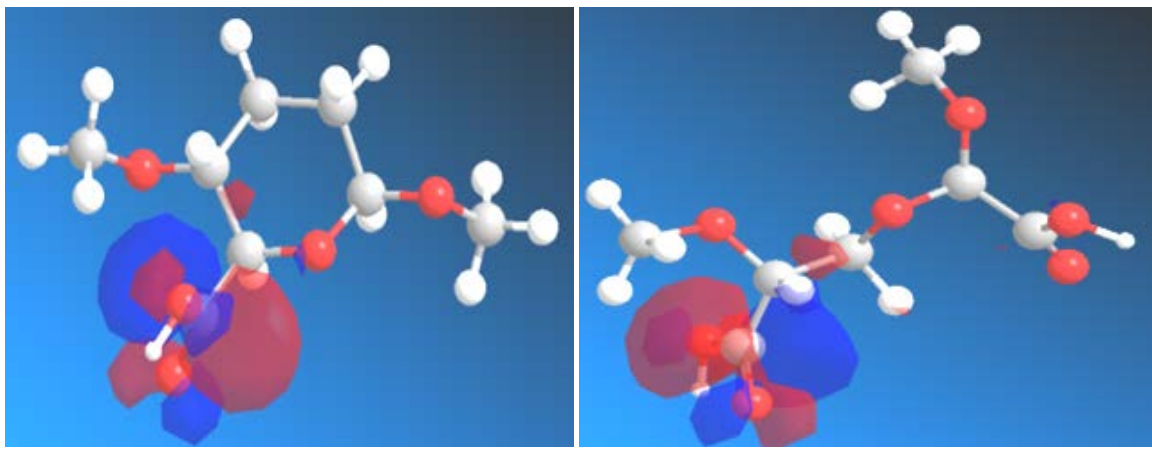


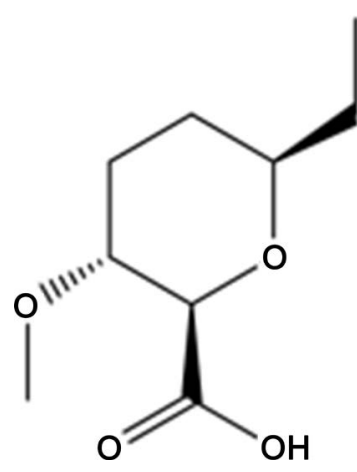

$\Delta \mathrm{E}=11,0$ эВ

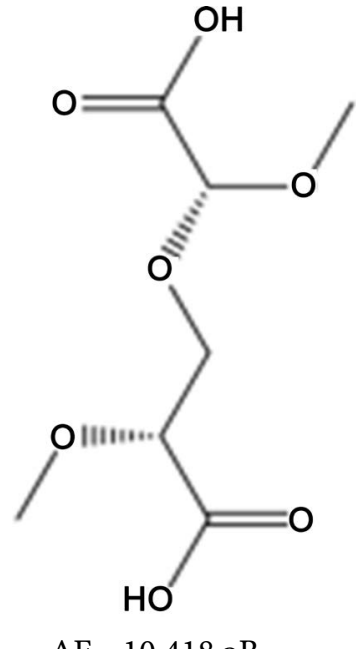

$\Delta \mathrm{E}=10,418$ эВ

Figure 8. HOMO and LUMO orbitals of the studied structures.

The contribution to the upper part of the valence band (HOMO) and the lower conduction band (LUMO) comes mainly from the $\mathrm{O} s / \mathrm{p}, \mathrm{C} \mathrm{s} / \mathrm{p}$ and $\mathrm{H} s$ states, respectively. As can be seen, LUMO orbitals in the cellulose molecule are distributed throughout the pyranose cycle, which indicates less reactivity with respect to the groups formed during chain breakage during hydrolysis, where the orbitals are localized on certain groups.

\section{Conclusions}

The possibility and optimal conditions for NC preparation and its stabilization were shown. The structure of the NC is proved by physical methods. Nanocellulose has rod-like shape with length of $50-300 \mathrm{~nm}$ and width of $10-40 \mathrm{~nm}$ with high crystallinity and spherical shape with low degree of crystallites and sizes of $50-300 \mathrm{~nm}$ is obtained depending on the synthesis conditions.

Thus, influence of condition of obtained NC on its properties and structures were investigated.

It was found that at mild conditions with using of physical factors (USD, MW) obtained NC has rod-like shape (whiskers) with sizes (50 - 300) $\times(10-40)$ $\mathrm{nm}$ with high degree of crystallinity and yield. Severe hydrolysis conditions lead to obtain NC like spherical shape (balls) with sizes by $(50-300) \mathrm{nm}$ low degree of crystallinity and less yield.

Quantum-chemical methods are used to calculate the electronic characteristics of cellulose and its hydrolysis products; the change in the difference between the energy of HOMO and LUMO is shown, which indicates a change in reactivity and the manifestation of specific properties.

\section{Conflicts of Interest}

The authors declare no conflicts of interest regarding the publication of this paper. 


\section{References}

[1] Habibi, Y., Lucia, L.A. and Rojas, O.J. (2010) Cellulose Nanocrystals Chemistry, Self-Assembly, and Applications. Chemical Reviews, 110, 3479-3500. https://doi.org/10.1021/cr900339w

[2] Ioelovich, M. and Figovsky, O. (2010) Structure and Properties of Nanoparticles Used in Paper Compositions. Mechanics Composite Materials, 46, 637-647. https://doi.org/10.1007/s11029-010-9160-Z

[3] Rebouillat, S. and Pla, F. (2013) State of the Art Manufacturing and Engineering of Nanocellulose: A Review of Available Data and Industrial Applications. Journal of Biomaterials and Nanobiotechnology, 4, 165-188. https://doi.org/10.4236/jbnb.2013.42022

[4] Lu, Y., Tekinalp, H.L., Eberle, C.C., Peter, W., Naskar, A.K. and Ozcan, S. (2014) Nanocellulose in Polymer Composites and Biomedical Application. Tappi Journal, 13, 47-54.

[5] Yuldoshov, Sh.A., Atakhanov, A.A., Sarymsakov, A.A. and Rashidova, S.Sh. (2015) Investigation of Reaction Activity of Cellulose and Its Products of Acid Hydrolysis. Macromolecules. An Indian Journal, 11, 51-57.

[6] Nazir, M.S., Wahjoedi, B.A., Yussof, A.W. and Abdullah, M.A. (2013) Eco-Friendly Extraction and Characterization of Cellulose from Oil Palm Empty Fruit Bunches. BioResource, 8, 2161-2172. https://doi.org/10.15376/biores.8.2.2161-2172

[7] Kong, W., Plant, T., Simonsen, J. and Evans, G. (2006) Cellulose Nanocrystal Electro-Optic Devices. Journal of Applied Physics, 97, Article ID: 053101.

[8] Wang, M.S. (2011) Surface Modification and Characterization of Nano Crystalline Cellulose. M.Sc. Sci. Thesis, Chalmers University of Technology, Goteborg.

[9] Moon, R.J., Martini, A., Nairn, J. and Simonsenf, J. (2011) Cellulose Nanomaterials Review: Structure, Properties and Nanocomposites. Chemical Society Reviews, 40, 3941-3994. https://doi.org/10.1039/c0cs00108b

[10] Elazzouzi-Hafraoui, S., Nishiyama, Y., Putaux, J.-L., Heux, L., Dubreuil, F. and Rochas, C. (2008) The Shape and Size Distribution of Crystalline Nanoparticles Prepared by Acid Hydrolysis of Native Cellulose. Biomacromolecules, 9, 57-65. https://doi.org/10.1021/bm700769p

[11] Zaini, L.H., Jonoobi, M., Tahir, P.Md. and Karimi, S. (2013) Isolation and Characterization of Cellulose Whiskers from Kenaf Bast Fibers. Journal of Biomaterials and Nanotechnology, 4, 37-44. https://doi.org/10.4236/jbnb.2013.41006

[12] Yu, M., Yang, R., Huang, L., Cao, X., Yang, F. and Liu, D. (2012) Preparation and Characterization of Bamboo Nanocrystalline Cellulose. Bioresources, 7, 1802-1812. https://doi.org/10.15376/biores.7.2.1802-1812

[13] Li, W., Wang, R. and Liu, S. (2011) Nanocrystalline Cellulose Prepared from Softwood Kraft Pulp via Ultrasonic-Assisted Acid Hydrolysis. BioResources, 6, 4271-4281.

[14] Janardhnan, S. and Sain, M.M. (2011) Targeted Disruption of Hydroxyl Chemistry and Crystallinity in Natural Fibers for the Isolation of Cellulose Nano-Fibers via Enzymatic Treatment. BioResources, 6, 1242-1250.

[15] Wang, Y., Wei, X., Li, J., Wang, F., Wang, Q. and Kong, L. (2013) Homogeneous Isolation of Nanocellulose by High Pressure Homogenization. Journal of Material Science and Chemical Engineering, 1, 49-52. https://doi.org/10.4236/msce.2013.15010

[16] Bondeson, D., Mathew, A. and Oksman, K. (2006) Optimization of the Isolation of Nanocrystals from Microcrystalline Cellulose by Acid Hydrolysis. Cellulose, 13, 
171-180. https://doi.org/10.1007/s10570-006-9061-4

[17] Segal, L., Creely, J.J., Martin, A.E. and Conrad, C.M. (1959) An Empirical Method for Estimating the Degree of Crystallinity of Native Cellulose Using the X-Ray Difractometer. Textile Research Journal, 29, 786-794.

https://doi.org/10.1177/004051755902901003 